\title{
HYALINISING TRABECULAR TUMOR- THYROID
}

C. Bharath, Vijayalaxmi Rampure

1. Professor \& HOD, Department of Pathology, Vijayanagara Institute of Medical Sciences. Bellary.

2. Assistant Professor, Department of Pathology, Mahadevappa Rampure Medical College, Gulbarga.

\section{CORRESPONDING AUTHOR}

Dr. C. Bharath

Vijayanagara Institute of Medical Sciences

Bellary.

E-mail: bhar5anu@yahoo.co.in

Ph: 00919483980702.

ABSTRACT: BACKGROUND: Hyalinising trabecular tumors of thyroid is an interesting but uncommon neoplasm of thyroid. This is presented because of its peculiar morphologic features, including encapsulation, trabecular pattern and marked hyalinization. OBJECTIVES: To know the morphologic feature and its histological behavior. METHOD: Hemithyroidectomy specimen was received with fixative. The specimen was grossed, bits were taken from appropriate areas \& processed in Histokinette, embedded in paraffin and the serial sections were stained with Hematoxylin \& Eosin. RESULTS: Histologically, showed encapsulated cellular growth, composed of follicular cells arranged in trabecular and follicular pattern. They are separated by fibrous bands and areas of abundant hyalinization seen. The nuclei of the cells were vesicular with indistinct nucleoli, having pale vacuolated cytoplasm. CONCLUSION: Hyalinizing trabecular tumor is an uncommon neoplasm with its peculiar morphologic features. It is sometimes mistaken for other neoplasm like- Papillary carcinoma, insular carcinoma \& paraganglioma.. Hence histologic evaluation of cytomorphologic features is important for definitive diagnosis.

KEYWORDS: Thyroid, hyalinization, trabecular pattern, neoplasm, histology.

INTRODUCTION: Hyalinising trabecular tumorof thyroid are interesting but uncommon neoplasm of follicular derivation with a histogenesisthat is still a debate.In 1987, Carney, et al ${ }^{1}$ first described a thyroid lesion with hyalinising patternof follicular derivation. The morphologic affinities between hyalinising trabecular tumor and papillary carcinoma, including nuclear pseudoinclusions and grooves, suggest that these tumor may be of similar origin ${ }^{2}$.These lesions has also been designated as paraganglioma like adenoma of thyroid by Brouner, et $\mathrm{al}^{3}$, because of its unusual histologic pattern.. Hence the importance lies in the fact that they are sometimes mistaken for other entities such as paraganglioma, medullary carcinoma and papillary carcinoma ${ }^{4}$.

CASE REPORT: A 35-year-old female presented with neck swelling since 8 years. On examination, revealed a solitary, oval to round smooth, firm swelling of $5 \times 3 \mathrm{cms}$ size, which moves deglutition and diagnosed clinically as solitary nodule thyroid. FNAC was done, which showed features of follicular neoplasm. Hemithyroidectomy was preformed and sent to laboratory.

PATHOLOGICAL FINDINGS: We received hemithyroidectomy specimen of $6.5 \times 3.5 \times 2 \mathrm{cms}$ in size. On gross examination, it was a well capsulated mass with congested vessels on surface. On 
section, heterogenous appearance was noticed with solid areas of grey-white and cytic area of $2.5 \mathrm{cms}$ in diameter, surrounded by grey-yellow rim of tissue (Fig.1).

Histologically, it was a well encapsulated cellular growth, composed of follicular cells arranged in trabeculaar and follicular pattern (Fig.3). The cells are separated by hyalinising fibrous bands. The central area of the tumor shows necrosis, also there are areas where follicles are seen separately within edematous hyalinisedstroma (Fig.2). The follicular cells have variable sized vesicular nuclei with indistinct nucleoli, occasional nuclei show grooving and pale vacuolated cytoplasm. There is no evidence for invasion or mitosis.

DISCUSSION: Hyalinising trabecular tumor are uncommon but intriguing neoplasm of the thyroid. Many are encapsulated and appear to behave in a benign fashion ${ }^{1}$.The designation hyalinising trabecular tumor has been accepted by the World Health Organization hand book on histologic typing of tumors of thyroid gland 5 . There remains concern, however that the tumor is a variant of papillary carcinoma, because of the overlap of its histologic and immunocytochemical profile of papillary carcinoma i.e., expression of high molecular weight keratins ${ }^{6,7}$. The benign behavior of most hyalinising trabecular tumor though is reassuring ${ }^{7}$. However some tumors are infiltrative, vasuloinvasive and metastasize to regional lymph nodes 8,9 .

The common presence in hyalinising trabecular tumor of morphologic feature traditionally is associated with papillary carcinoma, such as nuclear grooving, pseudoinclusions and psammoma bodies. Also the detection of in hyalinising trabecular tumor of RET/PTC mutations with a frequency similar to or even higher than that seen in papillary carcinoma ${ }^{2}$. Thus on the basis of this combined evidence, it has been suggested that hyalinising trabecular tumor is yet another variant of papillary carcinoma ${ }^{4,6}$.

Hyalinising trabecular tumor is the term given to a peculiar type of tumor exhibiting a prominent trabecular arrangement and equally prominent hyaline appearance ${ }^{10}$.

CONCLUSION: Hyalinising trabecular tumor of thyroid is an uncommon but potentially confusing neoplasm. It may be mistaken for other entities like papillary carcinoma, medullary carcinoma and also it should still be considered as a tumor with uncertain malignant potential. Further study with adequate follow up will continue to delineate the behavior and natural history of the lesion. Total thyroidectomy may be recommended for this lesion meeting the same criteria as papillary carcinoma and adequate histological examination is a must for a definitive diagnosis..

\section{REFERENCES:}

1. Carney JA, Ryan J \&GoellnerJR :Hyalinising trabecular adenoma of the thyroid gland. Americal journal of Surgical Pathology.1987; 11; 583 - 591.

2. Papotti, Mauro MD, Volante, et al: Hyalinising trabecular tumor of thyroid. American journal of Surgical Pathology. 2000; 24(2); 1615 - 1621.

3. Bronner MP, LivolsiVA, Jennings TA \& PLAT: Paraganglioma like adenomaa of thyroid gland, Surgical Pathology. 1988; 1; 383 - 389.

4. Cheung. Carol C MD, Boener, et al: Hyalinising trabecular tumor of thyroid - a variant of papillary carcinoma. American journal of Surgical Pathology. 2000; 24; 1622 - 1626. 
5. Hedinger C: Histological typing of thyroid tumors. WHO International Classification of Tumors, Berlin, Springer-Verlagi.1988.

6. Fenseca E, NeslandJM\&Sobrinho-Simoes M: Expression of stratified epithelial type cytokeratins in hyalinising trabecular adenoma supports this relationship with papillary carcinoma of thyroid. Histopathology. 1997; 31; 330 - 335.

7. Rothenberg, Harold J MD, Goellner, et al: Hyalinising trabecular adenoma of thyroid gland. American journal of Surgical Pathology. 1999; 23(1); 118 - 125.

8. McCluggage WG \& Sloan JM: Hyalinising trabecular adenoma of thyroid: morphology and differential diagnosis. Histopathology. 1996; 28; 357 - 362.

9. Molberg K \&Albores-Saavedea J: Hyalinising trabecular carcinoma of the thyroid gland. Human Pathology. 1994; 25; 192 - 197.

10. Juan Rosai: Hyalinising trabecular adenoma and related lesions. Ackerman's Surgical Pathology, Vol I, 9th edition, Mosby. 2004; $531-532$. .

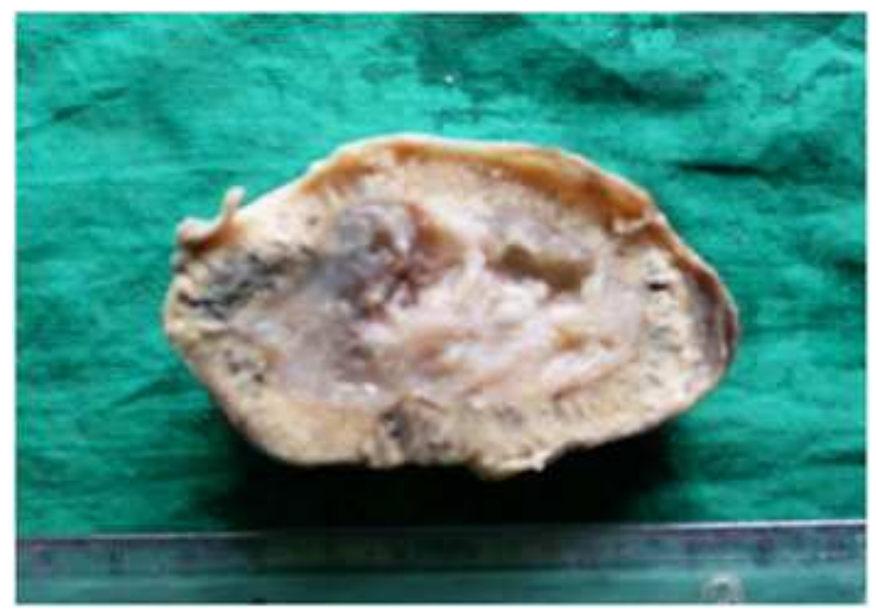

Fig.1. Sectioned thyroid, well capsulated showing grey white $\&$ cystic areas.

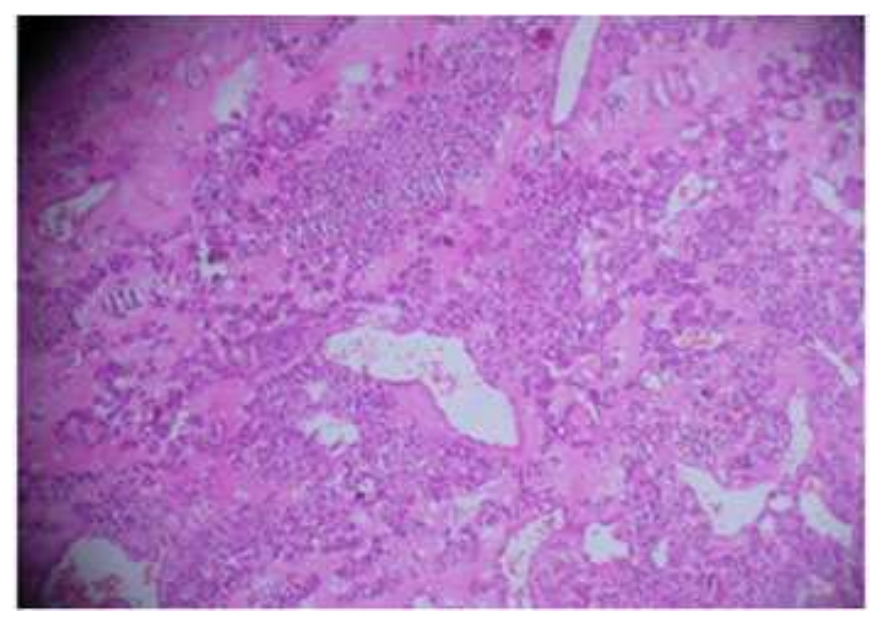

Fig.2.Histologically showing hyalinisedstroma\& follicle formation(H\&E.10x) 


\section{CASE REPORT}

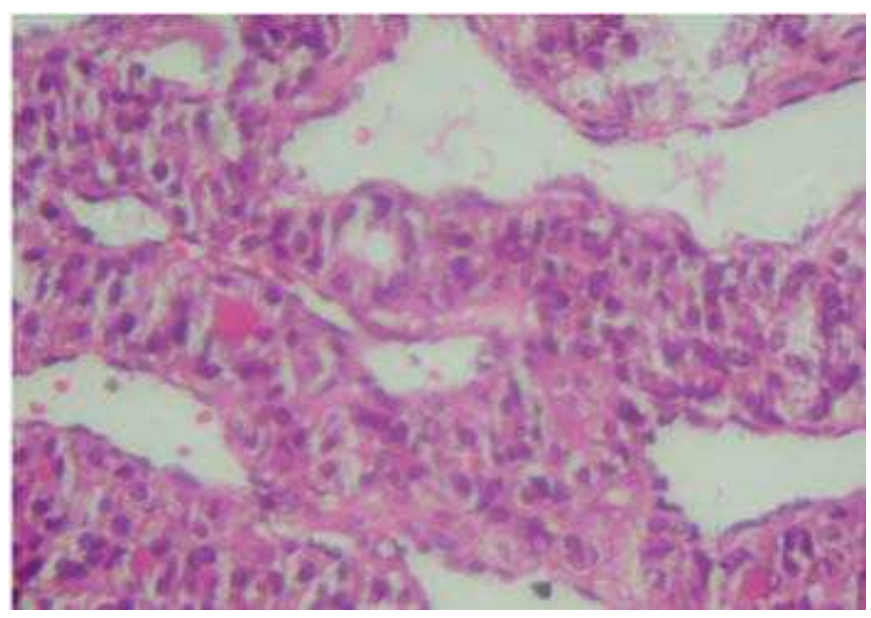

Fig.3.Histologically showing the trabecular structure by follicular cells(H\&E.45x) 